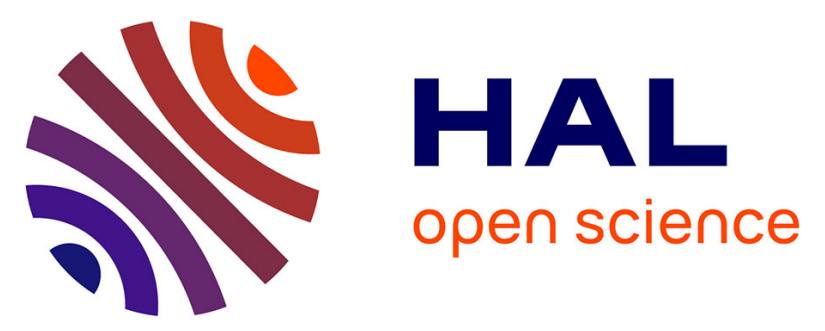

\title{
A methodology to quantify alterations in human upper limb movement during co-manipulation with an exoskeleton
}

Nathanaël Jarrassé, M. Tagliabue, J. Robertson, A Maiza, Vincent Crocher, Agnès Roby-Brami, Guillaume Morel

\section{To cite this version:}

Nathanaël Jarrassé, M. Tagliabue, J. Robertson, A Maiza, Vincent Crocher, et al.. A methodology to quantify alterations in human upper limb movement during co-manipulation with an exoskeleton. IEEE Transactions on Neural Systems and Rehabilitation Engineering, 2010, 18 (4), pp.389-397. 10.1109/TNSRE.2010.2056388 . hal-02110660

\section{HAL Id: hal-02110660 https://hal.science/hal-02110660}

Submitted on 25 Apr 2019

HAL is a multi-disciplinary open access archive for the deposit and dissemination of scientific research documents, whether they are published or not. The documents may come from teaching and research institutions in France or abroad, or from public or private research centers.
L'archive ouverte pluridisciplinaire HAL, est destinée au dépôt et à la diffusion de documents scientifiques de niveau recherche, publiés ou non, émanant des établissements d'enseignement et de recherche français ou étrangers, des laboratoires publics ou privés. 


\title{
A methodology to quantify alterations in human upper limb movement during co-manipulation with an exoskeleton
}

\author{
N. Jarrassé, M. Tagliabue, J. Robertson, A. Maiza, V. Crocher, A. Roby-Brami, G. Morel
}

\begin{abstract}
While a large number of robotic exoskeletons have been designed by research teams for rehabilitation, it remains rather difficult to analyse their ability to finely interact with a human limb: no performance indicators or general methodology to characterize this capacity really exist. This is particularly regretful at a time when robotics are becoming a recognized rehabilitation method and when complex problems such as 3D movement rehabilitation and joint rotation coordination are being addressed. The aim of this paper is to propose a general methodology to evaluate, through a reduced set of simple indicators, the ability of an exoskeleton to interact finely and in a controlled way with a human. The method involves measurement and recording of positions and forces during 3D point to point tasks. It is applied to a $4 \mathrm{DoF}$ limb exoskeleton by way of example.
\end{abstract}

Index Terms-Exoskeletons, rehabilitation, physical HumanRobot interaction (pHRi).

\section{INTRODUCTION}

\section{A. Context}

The potential of robotics for the rehabilitation of the upperlimb of patients with post stroke hemiparesis is now well recognized [1], [2], [3]. Robotic technology can enable early, intense and motivating rehabilitation and assist therapists who face a growing number of patients. Robot therapy, has been developing over the past decade [4] and is expected to bring benefits over traditional rehabilitation methods, mostly as a result of the large number of movement repetitions which can be provided by robotic devices [5]. However, recent clinical systematic reviews, including several robot designs, failed to demonstrate clear benefits of robot therapy over traditional rehabilitation methods [6], [7], [8]. Unquestionable proof of clinical benefit is still lacking in the literature. Most importantly to the robot designer, it is currently impossible

N. Jarrassé, V. Crocher and G. Morel are with the ISIR-UPMC (Institut des Systèmes Intelligents et de Robotique) - CNRS UMR 7222 from Université Pierre et Marie Curie - Paris6). 4 Place Jussieu 75252 Paris Cedex, France. (Phone: (33)146547812 , fax: (33)146547299; Email: \{crocher,jarrasse,morel\}@ isir.upmc.fr.)

M. Tagliabue is with CESeM (Centre d'Etude de la Sensorimotricité ) CNRS UMR 8194 - Université Paris Descartes, 45 rue des Saints Pères, 75270 Paris Cedex 06, France. (Phone: (33)142863315; fax: (33)142863399; Email: michele.tagliabue@parisdescartes.fr)

J. Robertson, A. Maiza and A. Roby Brami are with the LNP (Laboratory of Neurophysics and Physiology) - CNRS UMR 8119 - Université Paris Descartes, 45 rue des Saints Pères, 75270 Paris Cedex 06, France. (Phone: (33)142862138; fax: (33)149279062; Email: \{johanna.robertson,agnes.robybrami\}@univ-paris5.fr.)

Copyright (c) 2010 IEEE. Personal use of this material is permitted. However, permission to use this material for any other purposes must be obtained from the IEEE by sending a request to pubs-permissions@ieee.org. to map the robot design features and performances to its clinical effectiveness. Assessing the robot's performance is, by itself, a difficult problem due to the interactive nature of the device: simply listing engineer-world scores, such as maximum force, bandwidth or range of motion is of course informative regarding the robot's capacities, but it seems insufficient in the quantification of how well the device can interact with a subject.

Two important features can be identified to distinguish rehabilitation robots between each other in view of comparing their interaction capacities.

Considering first the mechanical design, manipulandum-type robots, based on the end effector approach in which the human-robot interaction occurs at the hand are to be distinguished from multi-contact devices, which present an interesting perspective for robot therapy. Exoskeletal and multi contact devices, have received growing interest in recent years, including in the field of rehabilitation [9]. The main differences between an exoskeleton and a manipulandum are the kinematic redundancy and the presence of multiple contact points between the device and the patient's limb. There are a number of exoskeletons under development for rehabilitation purpose with various objectives, joint designs and actuators (for a recent and exhaustive overview, see [10]). Three of them are under evaluation for shoulder-elbow coordination training in healthy subjects and stroke patients. The iPAM $3 \mathrm{D}$ rehabilitation robot is composed of two pneumatic robots controlling the forearm and the upper arm [11]. The ArMin robot [12] is a haptic robot with semi-exoskeleton kinematics with four active (shoulder and elbow) and two passive degrees of freedom. The T-Wrex is a passive device which provides gravity compensation [13], [14].

A second crucial characteristic of a rehabilitation robot is its interaction capacity. Indeed, rehabilitation robotics began by using robots to passively mobilize patients' limbs during the first stages of rehabilitation, when the patient is unable to move alone. However, the effectiveness of such passive movements for stimulating motor recovery is limited [15]. In order to stimulate motor recovery, it is essential for rehabilitation robots to exhibit finer interaction capabilities. Shared control of the movements must be possible as soon as the patient has recovered a minimal amount of motor capacity [16], [17].

The complex pHRI (physical Human-Robot Interaction) between an upper limb exoskeleton and its wearer reveals new as yet unstudied problems. Human motor control has particular biological characteristics due to the neural control of the 
multilink segments and the redundancy of the motor system as evidenced by kinematic regularities or invariances such as the smoothness of the endpoint kinematics [18] and the presence of inter-joint synergies [19]. The effect of human neural constrains on human robot interaction is only beginning to be explored [20] as well as the importance of humanrobot cooperative control for rehabilitation and training [21], [22], [23]. In addition, due to the fact that neuro-rehabilitation addresses issues relating to motor learning, the machines must allow patients to express whatever movement they can without hindering or suppressing any motor capability [24]. The great advantage of exoskeletons for rehabilitation is the possibility to control the segments of the upper limb and thus directly influence movement synergies, e.g. shoulder elbow coordination which is known to be perturbed following stroke [25]. Improving shoulder elbow coordination may improve function, thus impacting on the daily lives of patients [26]. It is thus very important that an exoskeleton should exhibit transparency. As has been suggested in [24], the robot must be able to 'get out of the way' if the patient is capable of making the movement. In this situation the robot is passive and thus must not perturb the patient's movement. Equally for evaluation purposes, if the robot is to measure the patient's movement capacity, it must influence the movement as little as possible.

In co-manipulation, transparency relates to the ability of the robotic system interacting with a human who is moving voluntarily, not to resist the intentional motion, and as such to allow natural, unperturbed movement. From an engineering point of view, this comes down to applying a null force and this is a good indicator of the ability of the system to generate precisely controlled forces. Indeed, any residual force that is applied to the subject's limb during voluntary movement will act as a bias when non zero forces need to be applied by the robot on the subject. Because human movement is often performed with low limb impedance/stiffness, it is very sensitive to perturbations [27]. Even small interaction forces could completely alter the coordination of the movement.

\section{B. Existing evaluation methods}

Despite the importance of a fine control of interaction between the patient and the robot in the rehabilitation context, there is a lack of performance indicators or methodology allowing objective evaluation of pHRIs, especially for multicontact devices [28]. Namely, apart from the evaluation of mechanical and control performances, which are generally available in the literature (see [10]), no generally adopted criteria exist to directly evaluate alterations in human movement parameters caused by the mechanics of the robot or its control limitations.

Mechanical approaches use the device bandwidth as a criterion for evaluating performance. If the bandwidth of the exoskeleton is smaller that of human movement, it is considered that robot will alter human movement or perception [9]. Other approaches consist of applying a load to the robot or fixing it to a subject and evaluating its ability to follow a trajectory. A reduced version of the IKO exoskeleton [29] was validated in this way using a PID driving a loaded robot forearm. Gopura et al [30], evaluated the capacity of a robot to displace the passive upper limb of health subjects. Kiguchi and Liyanage [31], evaluated co-manipulation tasks by assessing the effect of assistance provided by an exoskeleton during reaching tasks in healthy subjects. Rosen et al. [32] developed a more complex evaluation technique for their power amplifier structure including human arm moment and load moment, mechanical moment gain, mechanical work ratio and muscles activation ratio. However, none of these approaches include an experimental evaluation where scores are attributed to subjects performing tasks under robotic assistance. To the authors' knowledge, iPAM is the only multi-contact rehabilitation robot that has been evaluated through comparison between natural and assisted movements, [11]. The authors assessed peak deviation of the hand trajectory from a hypothetical ideal straight-line trajectory, the position of the hand in 3D and in the transverse plane and cyclograms of shoulder-elbow motion to conclude on the limited impact their robot has on subjects. We suggest that this approach is highly pertinent, because it takes into account the kinematic redundancy of humanrobot system due to the fact that the arm has more DoFs than those necessary to perform the reaching task. An analysis based solely on endpoint parameters could give an incomplete picture of the kinematic consequences of the pHRI. However, in addition to the kinematic redundancy taken into account by [11], a multi-contact human-robot system is also characterized by dynamic redundancy which must be considered. Indeed, the number of force and torque components which can be produced at each fixation point (6) is larger of those directly controlled by the device (4). Thus in order to fully investigate pHRIs, it appears very important to include an evaluation of performance in terms of all the force components. For all these reasons, we propose a methodology consisting of a three-level evaluation of human-robot interactions: endpoint trajectory generation, joint rotations and dynamic interaction. We chose to carry out this evaluation with the robot in transparent mode for the reasons described in the Context section. The evaluation consists of comparing simple pointing movements made by healthy subjects with and without the device connected to their limb, through a set of performance indices representing the three levels of analysis. This methodology is described in Section II, together with a simple experiment to obtain force and kinematic data. This data is used to calculate the different performance indicators. In Section III, we apply this methodology on healthy subjects with a 4 active degrees of freedom (DoF) arm exoskeleton. This exoskeleton, called ABLE, was developed with great attention paid to the reversibility (or mechanical transparency) issue [33]. Results are presented in Section IV and a discussion on the methodology is proposed in section V.

\section{Methodology}

\section{A. General description of the experiments}

We suggest that transparency should be evaluated in totally active mode: the subject performs the movement actively and the robot only follows without exerting active forces. In this 
mode, a robot can be qualified as transparent if the interaction forces applied on the human arm are null (or very low) and the trajectories appear kinematically equivalent to movements carried out without the robot.

We propose a comparison of movement parameters recorded during a simple pointing task performed by healthy subjects with and without the exoskeleton. Pointing movements are representative of movements used during rehabilitation sessions and are easy to setup and to analyze. It is important to position the targets in such a manner as to ensure that the subject has to explore different regions of the workspace, thus involving different joint rotations.

Another important aspect is to allow a few minutes of free training so that the subject can feel comfortable and safe with the device since initial movements may be perturbed by the neweness of the experience. A good indicator that the subject is ready to perform the experiment is when he/she feels safe and when the movements between two targets are qualitatively repeatable.

\section{B. Measurements}

We propose both an analysis of kinematics and of interaction forces between the robot and the subject.

1) Kinematic parameter measurements: A Codamotion ${ }^{\circledR}$ system was used to record the displacement of 6 optical markers placed on the subject's arm. For the calculation of joint angles, we chose the rigorous formalism of the ISB shoulder group [34]. Analysis was focused on shoulder elevation and elbow extension.

2) Force measurements: For force recording, we chose to fit the exoskeleton with one $\mathrm{F} / \mathrm{T}$ (Force/Torque) sensor per fixation point between human arm and the robot. These sensors have to be placed serially in the fixation mechanisms chain. Specific care has to be taken for the processing of F/T data. In order to evaluate equivalent moments applied by the robot on the arm segment, moment components read at the sensor center should be calculated at the center of the upper limb segment to which it is connected (see Fig.1): if $C$ is the sensor center and $G$ the upper limb segment center, with $\overrightarrow{C G}=d \vec{z}$, then moment at point $G$ shall be computed by:

$$
\overrightarrow{M_{G}}=\left[M_{x}, M_{y}, M_{z}\right]^{t}=\overrightarrow{M_{C}}-\overrightarrow{C G} \wedge \vec{R},
$$

with $\vec{R}=\left[F_{x}, F_{y}, F_{z}\right]^{t}$. In practice, $d$ is a constant distance estimated from where, on average, the upper limb segment is centered.

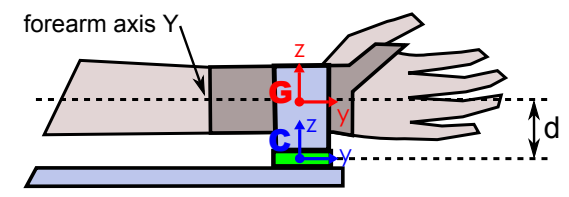

Fig. 1. Reconstructing forces and torques applied on limb from components read by the sensor

The sensors provide data on a set of force and torque components $\left(F_{x}, F_{y}, F_{z}, M_{x}, M_{y}, M_{z}\right)$ at each interaction point which will be used in the Performance Indices $(P I)$ presented below.

\section{Performance indices description}

1) Performance indices based on end-point trajectories: We chose to analyze 4 kinematic indices which inform us about different characteristics of the endpoint trajectory:

- $P I_{1}$ : Movement duration, simply corresponds to the time taken to carry out the required movement and gives an indication of the ease of moving under different conditions.

$$
P I_{1}=t_{\text {end }}-t_{\text {init }}
$$

where $t_{\text {init }}$ and $t_{\text {end }}$ have been identified as the first and the last frame, when the endpoint velocity is greater than $5 \%$ of the peak pointer velocity.

- $\mathrm{PI}_{2}$ : Velocity profile symmetry. Time of acceleration, divided by total movement time indicates how the velocity profile is altered. Perfect symmetry gives 0.5 , while if the index is small, more time is spent in deceleration, indicating possible problems with the regulation of inertia of the system,

$$
P I_{2}=\frac{t_{\text {acc }}-t_{\text {init }}}{t_{\text {end }}-t_{\text {init }}}
$$

where $t_{a c c}$ is the frame when the peak of velocity occurs.

- $\mathrm{PI}_{3}$ : Smoothness analysis. The jerk metric (average rate of change of acceleration during a movement) is defined as

$$
P I_{3}=\frac{1}{t_{\text {end }}-t_{\text {init }}} \int_{t_{\text {init }}}^{t_{\text {end }}}\left[\left(\frac{d^{3} x}{d t^{3}}\right)^{2}+\left(\frac{d^{3} y}{d t^{3}}\right)^{2}+\left(\frac{d^{3} z}{d t^{3}}\right)^{2}\right] d t
$$

where $[x, y, z]$ are the recorded endpoint (hand) coordinates. Large values for $\mathrm{PI}_{3}$ indicate that many corrections are made during the movement which would mean that the subject had difficulty in guiding the robot. To avoid an effect of execution time on this parameter, the trajectory of the hand was always resampled at 200 frames before computing the jerk.

- $\mathrm{PI}_{4}$ : Trajectory curvature. The maximum deflection of the hand path from a straight line joining the initial and final positions shows if the hand is deviated from its natural path. If a large deviation is observed during comanipulation with a robot, as compared to free motions, this suggests problems related to friction or inertia on particular axes or robot stiffness and damping structure problems.

$$
P I_{4}=\frac{\max \left(d_{p}(t)\right)}{\left\|\overrightarrow{P\left(t_{\text {end }}\right)}-\overline{P\left(t_{\text {in }}\right)}\right\|},
$$

where $d_{p}(t)=\frac{\left\|\left(\overrightarrow{P(t)}-\overrightarrow{P\left(t_{\text {in }}\right)}\right) \times\left(\overrightarrow{P\left(t_{\text {end }}\right)}-\overrightarrow{P\left(t_{\text {in }}\right)}\right)\right\|}{\left\|\overrightarrow{P\left(t_{\text {end }}\right)}-\overrightarrow{P\left(t_{\text {in }}\right)}\right\|} \|$ is the instantaneous distance of the vector position of the pointer, $\overrightarrow{P(t)}$, from the straight line joining $\overrightarrow{P\left(t_{i n}\right)}$ and $\overrightarrow{P\left(t_{\text {end }}\right)}$. 
2) Performance indices based on joint rotations: The YXY convention used produces angles for orientation of the elevation plane and shoulder internal/external rotation which are linked due to the two rotations around the same axis and cannot be separately interpreted in an anatomo-functional way [34]. Therefore, we will only focus on two angles in this paper: shoulder elevation and elbow extension.

- $P I_{5}$ : Final joint angles. The angles of the DoF calculated at the instant when the pointer touches the target. If angles differ from the no robot condition, this may indicate problems on particular axes.

- $\mathrm{PI}_{6}$ : Joint range of motion (R.O.M.). We calculated the maximum and minimum joint angles during the movement and subtracted the min from the max to obtain the total range of motion, this parameter gives an indication of joint motion throughout the movement.

- $P I_{7}$ : Cyclogram of the shoulder angular velocity according to elbow angular velocity. This index indicates alterations in joint synchronizations. These types of graphs are commonly used for posture coordination and gait analysis [35]. Cyclograms are generally created from angular positions, but we prefer to use angular velocity for several reasons. Firstly, velocity allows to bypass the reference problem of the positions. Namely, a velocity cyclogram always begins and ends at $(0,0)$. Secondly, it allows a simplified representation of the acceleration variations, and thus on smoothness of angular motion. Thirdly, velocity analysis allows to quickly evaluate the different movement phases because the velocity sign describes a flexion or an extension.

Note that indices PI5 and PI6 do not provide any intrinsic intersubject variability due to anatomical differences between subjects. Indeed, the subjects have to touch the targets with a pointer, the length of which was adjusted for every subject to maintain a constant distance between subject elbow and end-effector.

3) Performance indices based on interaction forces: Three indices were used to quantify the amount of force produced by the robot.

- $\mathrm{PI}_{8}$ : Force and moment averaged norms at the fixation. This index describes the amount of exchanged force at the points of interaction (distributed along the arm) averaged over the subjects and for each target.

$$
\left\{\begin{array}{c}
P I_{8 f}=\frac{\sum^{n}(\text { Mean }(\|\vec{F}\|))}{n} \\
P I_{8 t}=\frac{\sum(\text { Mean }(\|\vec{M}\|))}{n}
\end{array},\right.
$$

where $\|\vec{F}\|=\sqrt{F_{x}^{2}+F_{y}^{2}+F_{z}^{2}},\|\vec{M}\|=\sqrt{M_{x}^{2}+M_{y}^{2}+M_{z}^{2}}$ and $n$ is the number of subjects.

- PI9: Mean of each force and moment component absolute value for each sensor. This index allows a better understanding of the force interaction phenomena, by presenting the results along each axis. In accordance to the ISB convention, the $\mathrm{Y}$ axis of the sensor is oriented parallel to the human limb axis (the humerus axis for the arm and the ulna axis for the forearm) where as the $X$ and $\mathrm{Z}$ axes generate a transverse cut of the limb.

$$
P I_{9}=\sum(\operatorname{Mean}(|A|)) / n
$$

with $A \in\left\{F_{x}, F_{y}, F_{z}, M_{x}, M_{y}, M_{z}\right\}$.

\section{Statistical analysis}

Repeated measures ANOVA were carried out for each of the kinematic PIs with condition (robot / no robot), target (4 targets) and movement (5 repetitions) as independent factors. When significant effects were found, a Newman-Keuls post hoc test was applied in order to evaluate the effect of condition on each target. The results on the ANOVA will be presented both in terms of value of the probability distribution function, $F$, and $p$-value.

\section{Application to the evaluation of ABLE}

The proposed methodology was applied to evaluate an existing arm exoskeleton.

\section{A. ABLE, a 4 DoF upper limb exoskeleton}

ABLE (see Figure 2) is a 4 axis exoskeleton that has been designed by CEA-LIST based on innovative actuation technology [36]. Its degrees of freedom are composed of a spherical shoulder arrangement made with 3 coincident axes and a 1 DoF elbow pivot. The forearm is not actuated.
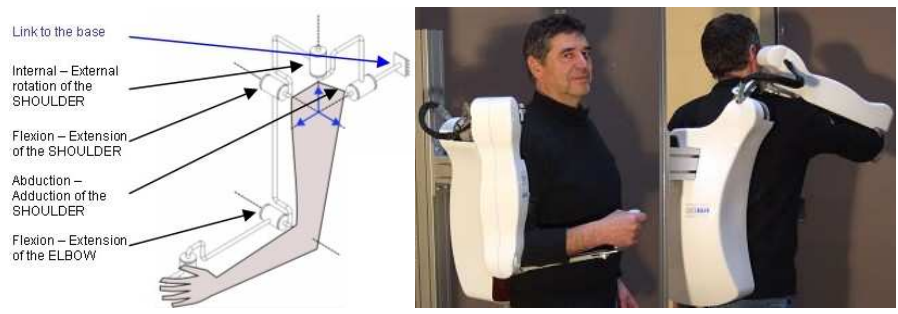

Fig. 2. ABLE 4 axis exoskeleton actuated by screw-and-cable actuators

ABLE DoFs are shown in Fig.2 and mechanical characteristics in the following table. Most of the robot's technological

\begin{tabular}{|l|c|c|c|c|}
\hline \multirow{2}{*}{ Joint } & Axis1 & Axis2 & Axis3 & Axis4 \\
\cline { 2 - 5 } & Abd/Add. & Int/Ext Rot. & Flex/Ext. & Flex/Ext. \\
\cline { 2 - 5 } & \multicolumn{4}{|c|}{ Shoulder } \\
\hline Amplitude & \multicolumn{4}{|c|}{ Dlbow deg. } \\
\hline Motors & \multicolumn{4}{|c|}{ DC Faull-Screw and Cable (SCS) } \\
\hline Transmissions & \multicolumn{4}{|c|}{$>1 \mathrm{~m} / \mathrm{s}$} \\
\hline Speed & $18 \mathrm{Nm}$ & $18 \mathrm{Nm}$ & $13 \mathrm{Nm}$ & $13 \mathrm{Nm}$ \\
\hline Joint torque & $50 \mathrm{~N}$ & $50 \mathrm{~N}$ & $50 \mathrm{~N}$ & $50 \mathrm{~N}$ \\
\hline Equiv. effort in hand & &
\end{tabular}

originality comes from its actuation and transmission system, which is based on a patented Screw-and-Cable system (SCS) [33]. The hardware characteristic of ABLE makes it an excellent platform for physical rehabilitation therapies. Its naturally compliant joints ensure safety when using the robot for patients with physical disability. 


\section{B. Experimental Setup}

1) Device control: It is essential to make the ABLE as transparent as possible, in order to limit the residual force level, which may appear due to hyperstaticity, inertia and friction. As ABLE is only fitted with optical encoders, we do not have access to an acceleration signal. The transparency is thus achieved by an experimentally identified gravity compensation for all axes. The robot controller architecture is based on a PC104 board with two endowed 3 channel axis controllers. It runs the compensation open loop control law at $1 \mathrm{kHz}$ using a real time operating system (RTlinux).

The controller was also fitted with two Analog and Digital I/O PCI cards (a National Instrument model 6034E and a Sensory 526) which were used for acquiring the readings of the two $\mathrm{F} / \mathrm{T}$ sensors during the movement every millisecond. Note that $\mathrm{F} / \mathrm{T}$ data is not used in real time at the controller level, because they are installed only during the characterization phase.

2) Task: Ten voluntary subjects were involved in this experiment. In order to exploit the robot's DoFs, pointing movements were made in a standing position to four targets positioned in different parts of the work space allowing us to analyze the interactions between the subject and the robot when different axes of motion were involved. Three lines were drawn from the starting position, one in the para-sagittal plane and the others at $45^{\circ}$ either side of the first line. The targets were marked on poles which were placed $50 \mathrm{~cm}$ from the starting position on each of the three lines. The target height was positioned at the level of the exoskeleton elbow axis for targets 1-3 and target 4 was positioned above target 2, the height was equal to the horizontal distance between targets 1-2 and 2-3 (see Fig 3 and 4).

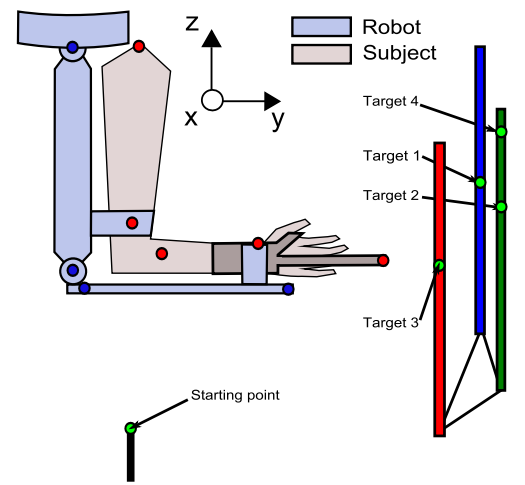

Fig. 3. Schematic of the Codamotion ${ }^{\circledR}$ markers positions during the experiments (In red, markers on the arm, in blue markers on the robot and green markers on the targets)

The starting point was standardized with the elbow in maximum extension, the humerus vertical and the forearm in mid prone position. The subjects rested their backs against the support of the robot and a large belt was used to prevent trunk movement and a splint was used to prevent wrist motion, both of which would confound analysis of shoulder and elbow angles. A pointer was fixed to the splint.

Ten healthy volunteers naive to the study were included ( 9 male and 1 female). They were all aged between 22-30. They gave informed consent according to ethical procedures.
A Codamotion ${ }^{\circledR}$ system was used to record (200 Hz sampling rate) the position of active markers placed on the subject and also on the robot (for data control) as shown in figure 3. Only results relative to the subjects will be described here.

Two conditions were tested: without robot (subjects made movements without wearing the exoskeleton) and with robot (subjects made movements with the exoskeleton). Subjects were allowed to practice moving with the robot for 5 minutes prior to recording. Five movements were recorded to each target. Subjects were instructed to move as naturally as possible to touch the target.
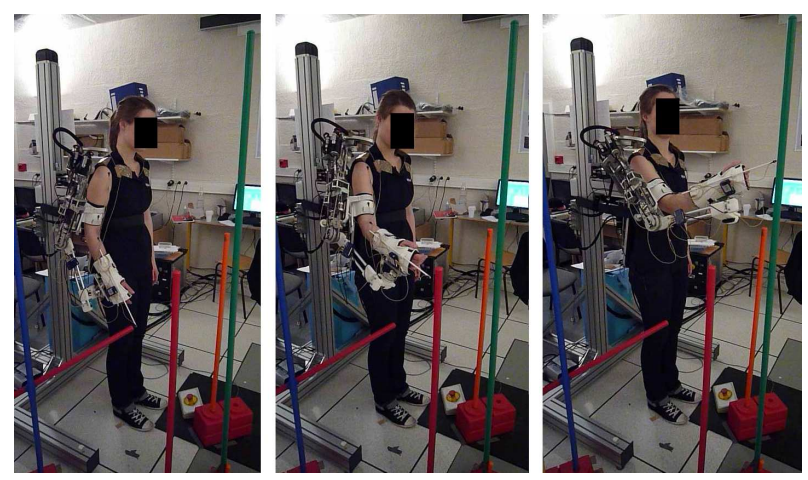

Fig. 4. Subject pointing to different targets wearing exoskeleton.

\section{Results}

\section{A. End-point analysis: $P I_{1}$ to $P I_{4}$}

For the calculation of kinematic parameters relating to the hand trajectory, the 3D trajectory of the markers was smoothed using a zero-phase forward and reverse digital filter at $5 \mathrm{~Hz}$. This reduced noise related to the recording system.
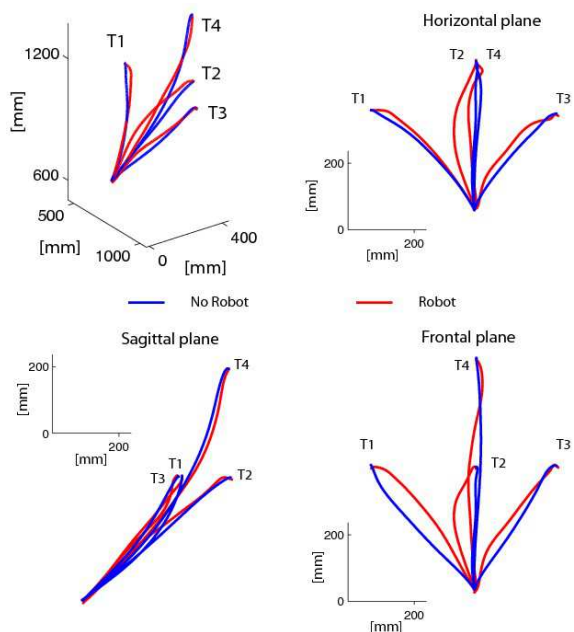

Fig. 5. Trajectory of the pointer for subject 1 in the three conditions (the three movement repetitions have been averaged). The blue line represents the "No robot" condition, the red "With the robot" one

Visualization of the path of the endpoint under the different conditions gives, in Fig. 5, a qualitative impression of the effect of the robot on the human movement. It is obvious that the interaction with the robot deviates the hand from its 
natural path particularly in the horizontal and frontal planes. From the results in Fig.6, that are averaged over the 10 subjects and the five repetitions, it is evident that the robot alters the kinematics of the hand trajectory. $P I_{1}$ : execution time is longer with the robot $(F(1,9)=27.59, p<0.01)$. $P I_{2}$ indicates that the deceleration phase was proportionally longer with the robot suggesting that the movement was affected by additional inertia and perhaps the friction was overcompensated $(F(1,9)=30.16, p<0.01) . P I_{3}$ shows that endpoint path tended to be more curved for Targets 1 to 3 with the robot as shown in Fig 5, and less curved for Target 4, but no statistically significant effects were found. Finally, $P_{4}$ indicates that jerk was significantly higher with the robot $(F(1,9)=17.73, p<0.01)$ suggesting that the subject made more movement corrections in this condition. For each of these PIs the effect of the robot interaction was similar for all targets.
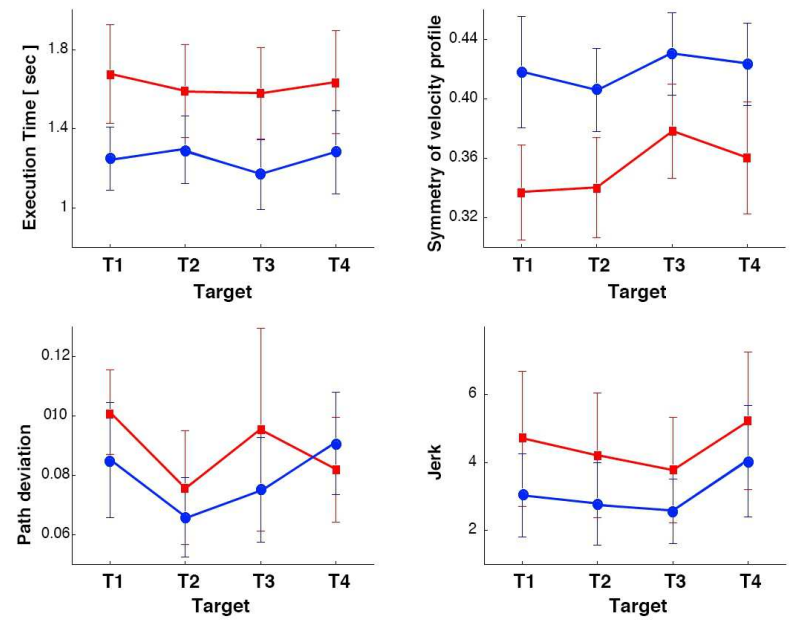

Fig. 6. $P I_{1}$ to $\mathrm{PI}_{4}$ : Mean and standard deviations for the 4 kinematic PIs related to pointer trajectory. (Red: with the robot; Blue: without)

\section{B. Joint rotations analysis: $\mathrm{PI}_{5}$ to $\mathrm{PI}_{7}$}

1) $\mathrm{PI}_{5}$ and $\mathrm{PI}_{6}$ : Final joint angles and Angular range of motion: As illustrated in Fig. 7, both range of elbow motion $(F(1,9)=17.8, p<0.01)$ and shoulder $\operatorname{motion}(F(1,9)=$ $72.9, p<0.01)$ were increased in the robot versus no robot condition.

Post hoc tests showed this was significant for elbow extension for target 2 and for shoulder elevation for targets 2-4 suggesting that the robot particularly carries the upper arm segment away from its natural trajectory. Analysis of final angles showed that the elbow was significantly less extended $(F(1,9)=21.4, p<0.01)$ and the shoulder more elevated $(F(1,9)=17.2, p<0.01)$ in the robot condition compared with the no robot condition although post hoc testing showed this was only significant for elbow extension for target 1 .

2) $\mathrm{PI}_{7}$ : Cyclogram of the shoulder angular velocity according to elbow angular velocity: Fig. 8 presents the velocity cyclogram during movement to the targets of one subject. There exists several ways to analyze such a graph: if the overall shape is preserved but there is a scale factor between the two conditions, then articular coordination is preserved but
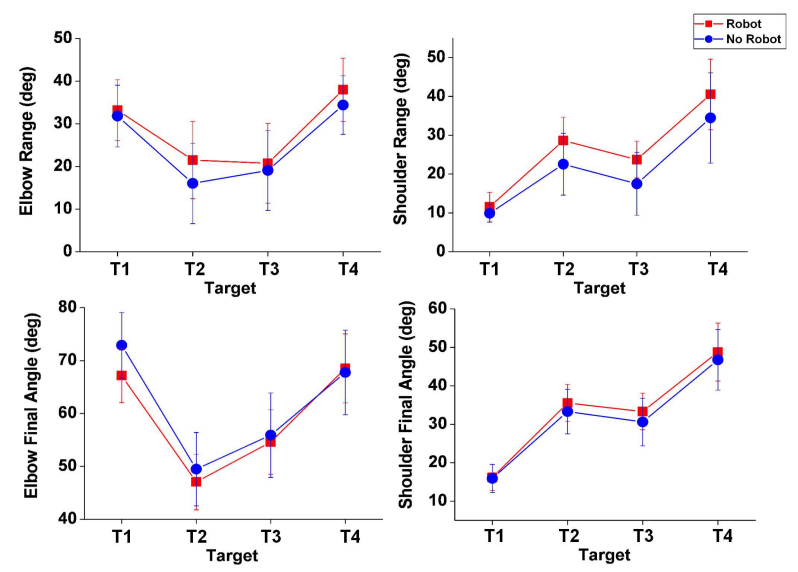

Fig. 7. $P I_{5}$ and $P I_{6}$ : Final joint angles and Angular range of motion for the two considered angles (shoulder elevation and elbow extension).Red: with the robot; Blue: without

velocity is altered (movement with robot is faster or slower). A drift of the cyclogram from one of the four quadrants to
Target 1
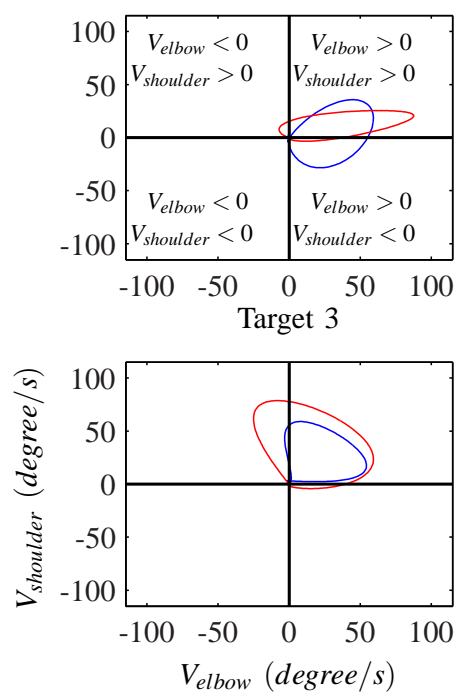

Target 2
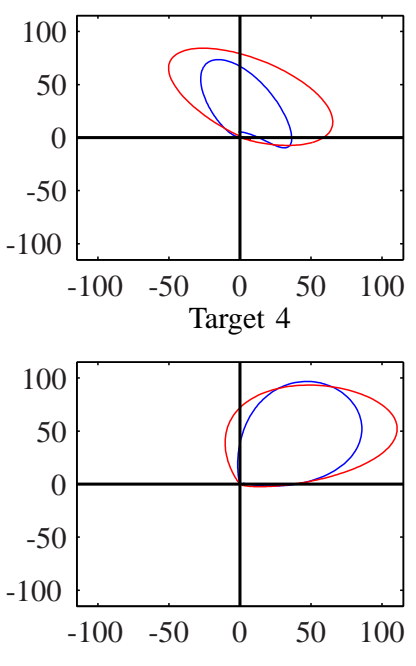

Fig. 8. $P I_{7}$ : Example of one subject velocity cyclogram during movement to the four targets (Blue: without robot; Red: with robot)

another indicates a change in the movement and a new phase relationship. In the example given here, during the movement to target 3, even if synchronization seemed to be preserved, a bigger flexion (negative elbow velocity) indicates a higher segmentation of the movement.

\section{Interaction forces analysis $\mathrm{PI}_{8}$ and $P \mathrm{I}_{9}$}

Analysis of this parameter is obviously limited by the fact that it can only be measured in the robot condition. However, it provides a quantification of the general residual, undesired and uncontrolled forces acting between the subject and the robot. Indeed the experiments are conducted with a robot in an active mode, and theoretically no forces should be applied on the upper limb on the component controlled by the robot. 
Fig. 9 indicates the overall force level for every target averaged over the ten subjects which appears higher on the forearm (up to $12 \mathrm{~N}$ and $6 \mathrm{~N} . \mathrm{cm}$ ) than on the upper arm (up to $8 \mathrm{~N}$ and

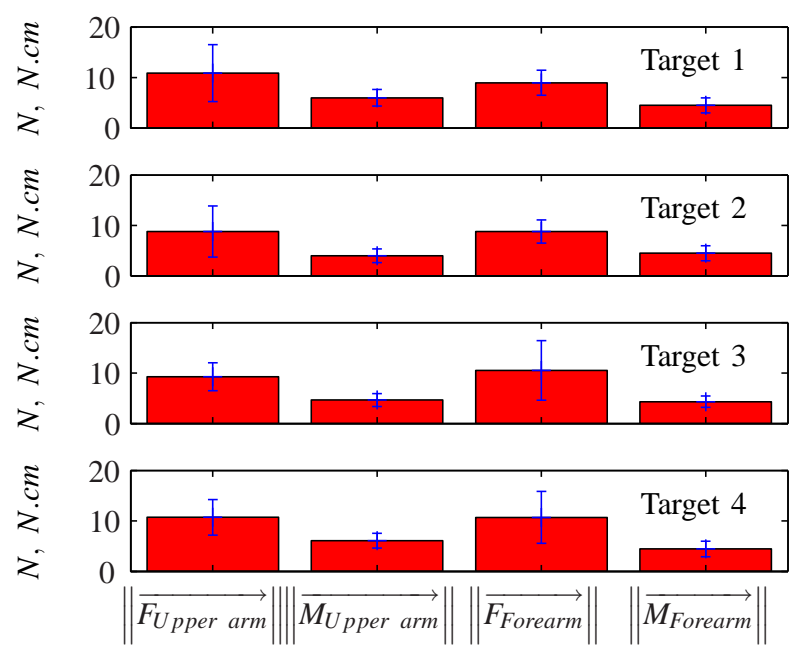

Fig. 9. $P I_{8}$ : Forces/Moments average norm on the two fixations for the 4 targets(Mean on 10 subjects)

6 N.cm).

It is important to note that, since the low level robot controller is designed to apply a zero torque, all the forces that appear arise from unmodelled phenomenons: robot joint friction, robot inertia and interaction at the fixation devices due to hyperstaticity and deformations induced by kinematic discrepancies between the robot and arm kinematics. All these effects are rather difficult to estimate a priori which justifies conducting comanipulation experiments for quantification purposes.

The components decomposition presented in Fig. 10, allows a better understanding of the physical interaction phenomena.

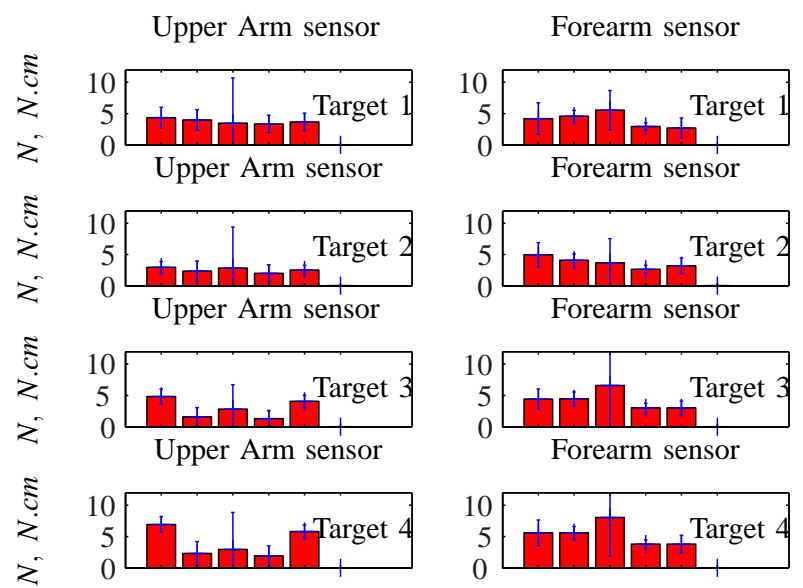

Fig. 10. $P I_{9}$ : Mean of each force and moment absolute value for the upper arm and forearm sensors (Mean on 10 subjects)

Indeed $F_{x}$ and $F_{z}$ are the forces transmitted from the subject in order to displace the robot whereas $F_{y}, M_{x}, M_{y}$ and $M_{z}$ are not compatible with the robot motion and thus arise from hyperstaticity. Figure 10, for example, indicates a high force level for the $F_{y}$ component (along the upper arm axis), which is a typical problem due to the inability to adjust the exoskeleton limb lengths to the human limbs.

\section{Discussion}

In this paper we have proposed a methodology for the evaluation of pHRI, which we applied to the example of a 4 DoF exoskeleton. This work was a pilot study which aimed, not to quantify the perturbations induced by a specific exoskeleton, but to focus on the necessity of using a rigorous methodology to gain a deeper understanding of physical human-robot interaction. In particular, the proposed methodology aims at evaluating the effect of interaction with a multi-contact robot at three different levels of human motor performance: endpoint trajectory generation, joint rotations and dynamic interactions. This required the use of a reference condition without robot in order to quantify alterations in movement parameters caused by the robot. The method can easily be transferred to different types of multi-contact robotic devices in order to evaluate pHRI and to quantify changes in performance when control laws are improved or mechanical changes are made. However, for different robotic applications such as orthopedic rehabilitation, different PI's may be relevant.

The results obtained by using a variety of PIs for each level of evaluation support our multiple approach to the evaluation of multi-contact robotic devices.

Our findings highlight the complexity of these interactions and the necessity of analyzing a variety of parameters. Different conclusions could have been obtained from each of the individual parameters. For example, endpoint movement duration was increased with the robot, suggesting a slowing down of the whole movement. On the other hand, joint angular velocities were increased with the robot (See Fig. 8) along with the joint range of motion, indicating that the slowing of the endpoint movement was not related to a general hindering of motion by the robot. Similarly while analysis of the endpoint and joint rotation parameters could have lead to the conclusion that movement alterations were due to friction-inertia phenomena or to the action of the device motors, the analysis of the individual interaction force components (See Fig. 10) shows that kinematic alterations are also likely to be due to hyperstaticity and misalignment of subject-robot joint axes.

The analysis of interaction forces and whole-limb kinematics is fundamental when the robot is to be used for rehabilitation purposes. Training movements with a non transparent robot is likely to perturb the motor coordination of the patient, not only from a biomechanical point of view, but also through mechanisms of sensory-motor adaptation. The sensory feedback produced when the patient moves while wearing the exoskeleton could induce changes in sensorymotor maps resulting in unnatural motor strategies. Indeed, the neurologically-impaired patients for whom these types of robots are conceived have sensory-motor integration deficits. It is thus particularly important to minimize external interferences which could influence motor learning processes. 
In the future, the methodology described here will be used to evaluate the quality of human-robot interactions in the framework of iterative prototyping of the robot and of its command laws. In particular, we recently developed a new system of fixations allowing the release of some degrees of freedom in order to reduce hyperstaticity and to adapt the robot to the size of the human subject. The present method will be used to test whether this new system improves the quality of human-robot interactions. In addition, innovative command laws directed to the control of joint rotations are under development in order to target rehabilitation exercises for hemiparetic patients, focusing on shoulderelbow coordination. The present method will be used to evaluate the effects of these new commands in healthy human subjects and perform fine adjustments before investigating the concept in hemiparetic patients. It would be also interesting to correlate the quantifiable improvement of the different PI with the actual changes in the subject perception of the robot. This study would allows to focus further technological development on the interaction aspects which appear to be more critical for the confort of the robot users. Finally, this method could be easily adapted to other exoskeletons in order to gather comparative data on the human-robot interactions with different robotic structures.

\section{ACKNOWLEDGMENT}

This work is supported by the national research agency ANR PSIROB 2006, through the BRAHMA (BioRobotics for Assisting Human Manipulation) project (ANR-06-ROBO0003) and by the CNRS (Centre National de la Recherche Scientifique) project AIDER (Analyse des Interactions Dynamiques avec un Exosquelette pour la Réhabilitation). Agnès Roby-Brami is supported by INSERM.

\section{REFERENCES}

[1] R. Colombo, F. Pisano, S. Micera, A. Mazzone, C. Delconte, M. C. Carrozza, P. Dario, and G. Minuco. Robotic techniques for upper limb evaluation and rehabilitation of stroke patients. In IEEE Trans. Neural Syst. Rehabil. Eng., volume 13, pages 311-324, 2005.

[2] W. S. Harwin, J. L. Patton, and V. R. Edgerton. Challenges and opportunities for robot-mediated neurorehabilitation. In Proc. of the IEEE, volume 94, pages 1717-1726, Sept. 2006.

[3] B. T. Volpe, P. T. Huerta, J. L. Zipse, A. Rykman, D.Edwards, L. Dipietro, N. Hogan, and H. I. Krebs. Robotic devices as therapeutic and diagnostic tools for stroke recovery. Archives of Neurology, 66(9):10861090, September 2009.

[4] H. I. Krebs, N. Hogan, M. L. Aisen, and B. T. Volpe. Robot-aided neurorehabilitation. IEEE Trans. Rehabil. Eng., 6:75-87, Mar 1998.

[5] B. T. Volpe, D. Lynch, A. Rykman-Berland, M. Ferraro, M. Galgano, N. Hogan, and H. I. Krebs. Intensive sensorimotor arm training mediated by therapist or robot improves hemiparesis in patients with chronic stroke. 22(3):305-310, 2008.

[6] G. B Prange, M. J. A. Jannink, C. G. M. Groothuis-Oudshoorn, H. J. Hermens, and M. J. Ijzerman. Systematic review of the effect of robotaided therapy on recovery of the hemiparetic arm after stroke. Journal of Rehabilitation Research and Development, 43(2):171-184, April 2006. PMID: 16847784

[7] G. Kwakkel, B. J. Kollen, and H. I. Krebs. Effects of Robot-Assisted therapy on upper limb recovery after stroke: A systematic review. Neurorehabil Neural Repair, 22(2):111-121, April 2008.

[8] J. Mehrholz, T. Platz, J. Kugler, and M. Pohl. Electromechanical and Robot-Assisted arm training for improving arm function and activities of daily living after stroke. Stroke, pages 392-393, March 2009

[9] J. C. Perry, J. Rosen, and S. Burns. Upper-limb powered exoskeleton design. IEEE/ASME Trans. Mechatronics, 12:408-417, Aug 2007.
[10] R. A. R. C. Gopura and K. Kiguchi. Mechanical designs of active upper-limb exoskeleton robots: State-of-the-art and design difficulties. In Rehabilitation Robotics, 2009. ICORR 2009. IEEE Intl. Conf. on, pages 178-187, june 2009 .

[11] A. Jackson, P. Culmer, S. Makower, M. Levesley, R. Richardson, A. Cozens, M. M. Williams, and B. Bhakta. Initial patient testing of ipam-a robotic system for stroke rehabilitation. In Rehabilitation Robotics, 2007. ICORR 2007. IEEE 10th Intl. Conf. on, pages 250 256, june 2007.

[12] T. Nef, M. Guidali, and R. Riener. ARMin III - arm therapy exoskeleton with an ergonomic shoulder actuation. Applied Bionics and Biomechanics, 6(2):127, 2009.

[13] R.J. Sanchez, J. Liu, S. Rao, P. Shah, R. Smith, T. Rahman, S. C. Cramer, J. E. Bobrow, and D. J. Reinkensmeyer. Automating arm movement training following severe stroke: functional exercises with quantitative feedback in a gravity-reduced environment. IEEE Trans. Neural Syst. Rehabil. Eng., 14(3):378-389, September 2006.

[14] S.J. Housman, K.M. Scott, and D.J. Reinkensmeyer. A randomized controlled trial of Gravity-Supported, Computer-Enhanced arm exercise for individuals with severe hemiparesis. Neurorehabil Neural Repair, February 2009.

[15] D. Lynch, M. Ferraro, J. Krol, C. M. Trudell, P. Christos, and B. T. Volpe. Continuous passive motion improves shoulder joint integrity following stroke. Clinical Rehabilitation, 19(6):594-599, 2005.

[16] N. Hogan.and H. I. Krebs., B. Rohrer, J. Palazzolo, J. L. Dipietro., S. E. Fasoli, J. Stein, R. Hughes, W. R. Frontera, D. Lynch, and B. T. Volpe. Motions or muscles? some behavioral factors underlying robotic assistance of motor recovery. J Rehabil. Res. Dev., 43(5):605-618, 2006.

[17] J. L. Patton and F. A. Mussa-Ivaldi. Robot assisted adaptative training: Custom force fields for teaching movement patterns. IEEE Rev. Biomed. Eng., 51:636-646, 2002.

[18] T. Flash and N. Hogan. The coordination of arm movements: an experimentally confirmed mathematical model. J. Neurosci., 5(7):16881703, 1985.

[19] M.L. Latash, J.P. Scholz, and G. Schner. Toward a new theory of motor synergies. Motor Control, 11(3):276-308, July 2007.

[20] D. Campolo, D. Accoto, D. Formica, and E. Guglielmelli. Intrinsic constraints of neural origin: assessment and application to rehabilitation robotics. IEEE Trans. Robot., 25(3):492-501, 2009.

[21] L. E. Kahn, W. Z. Rymer, and D. J. Reinkensmeyer. Adaptive assistance for guided force training in chronic stroke. Engineering in Medicine and Biology Society, 2004. IEMBS '04. 26th Annual Intl. Conf. of the IEEE, 1:2722-2725, Sept. 2004.

[22] L. Marchal-Crespo and D. J. Reinkensmeyer. Review of control strategies for robotic movement training after neurologic injury. Journal of NeuroEngineering and Rehabilitation, 6:20-20.

[23] M. Matja1, N. Tobias1, and R. Robert1. A novel paradigm for patientcooperative control of upper-limb rehabilitation robots. Advanced Robotics, (8):843-867(25), 2007.

[24] N. Hogan and H. I. Krebs. Interactive robots for neuro-rehabilitation. Restorative Neurology and Neuroscience, 22(3-5):349-358, 2004. PMID: 15502275.

[25] M. C. Cirstea, A. B. Mitnitski, A. G. Feldman, and M. F. Levin. Interjoint coordination dynamics during reaching in stroke. Experimental Brain Research, 151(3):289-300, 2003.

[26] S. M. Michaelsen, R. Dannenbaum, and M. F. Levin. Task-specific training with trunk restraint on arm recovery in stroke: randomized control trial. Stroke, 37, 2006.

[27] H. Gomi and M. Kawato. Human arm stiffness and equilibrium-point trajectory during multi-joint movement. Biological Cybernetics, 1997.

[28] R. Alami, A. Albu-Schaeffer, A. Bicchi, R. Bischoff, R. Chatila, et al. Safe and dependable physical human-robot interaction in anthropic domains: state of the art and challenges. In Intelligent Robots and Systems, 2006. IROS 2006, IEEE/RSJ Int. Conf. on, pages 1753-1759.

[29] F. Martinez, I. Retolaza, A. Pujana-Arrese, A. Cenitagoya, J. Basurko, and J. Landaluze. Iko:esign of a five actuated dof upper limb exoskeleton oriented to workplace help. In Biomedical Robotics and Biomechatronics, 2008. BioRob 2008. 2nd IEEE RAS EMBS Intl. Conf. on, Oct. 2008.

[30] R.A.R.C. Gopura and K. Kiguchi. Development of a 6dof exoskeleton robot for human upper-limb motion assist. In Information and Automation for Sustainability, 2008. ICIAFS 2008. 4th Intl. Conf. on, Dec. 2008.

[31] K. Kiguchi and M. Liyanage. Study of a 4dof upper-limb power-assist intelligent exoskeleton with visual information for perception-assist. In Robotics and Automation, 2008. ICRA 2008. IEEE Intl. Conf. on, pages 3666-3671, 2008 
[32] J. Rosen, M. Brand, M. B. Fuchs, and M. Arcan. A myosignal-based powered exoskeleton system. IEEE Trans. Syst., Man, Cybern., pages 210-222, 2001.

[33] P. Garrec, J. P. Martins, and J. P. Friconneau. A new technology for portable exoskeletons. AMSE2004, Journal of the Association for the Advancement of Modelling, 65, 2004.

[34] G. Wu, F. C. van der Helm, H. E. Veeger, M. Makhsous, P. Van Roy, C. Anglin, J. Nagels, A. R. Karduna, K. McQuade, X. Wang, F. W. Werner, and B. Buchholz. Isb recommendation on definitions of joint coordinate systems of various joints for the reporting of human joint motion-part ii: shoulder, elbow, wrist and hand. Journal of Biomechanics, 35:543-548, May 2005.

[35] T. Krasovsky and M. F. Levin. Review: Toward a better understanding of coordination in healthy and poststroke gait. Neurorehabil Neural Repair, 24(3):213-224, March 2010.

[36] P. Garrec, J. P. Friconneau, Y. Measson, and Y. Perrot. Able, an innovative transparent exoskeleton for the upper-limb. Intelligent Robots and Systems, 2008. IROS 2008. IEEE/RSJ Intl. Conf. on, pages 14831488, Sept. 2008.

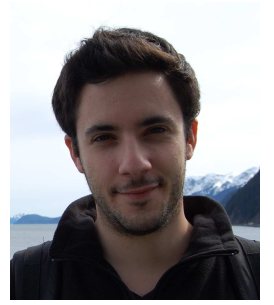

Nathanael Jarrassé received his degree in Industrial Systems Engineering from the Ecole Nationale Supérieure d'Arts et Métiers (ENSAM) and a Master of Science degree in Mechanics and System Engineering in 2006 from the Univ. P\&M Curie, France. He is currently finishing his Ph.D. at the ISIR-UPMC laboratory, and works on the control of an exoskeleton for assisting motor rehabilitation. His work in these areas focuses on kinetostatic analysis, physical Human-Robot interaction, movement analysis and transparency.

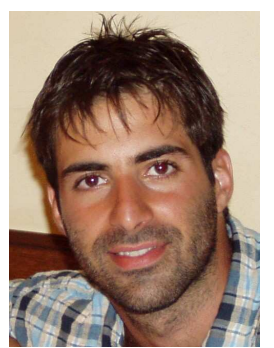

Michele Tagliabue received the M.S. in biomedical engineering at the Politecnico di Milano, Italy, in 2002, and the European Ph.D in Bioengineering at the Inter-Polytechnic excellence Ph.D School, Italy, in 2006. His research interests include human motor control, biomechanics and sensory-motor integration mechanisms. In particular, he developed special skill in the field of microgravity experiments as well as in clinical studies involving patients with Parkinson's disease. He likes to conceive innovative experimencaption, virtual reality systems and robotic devices.

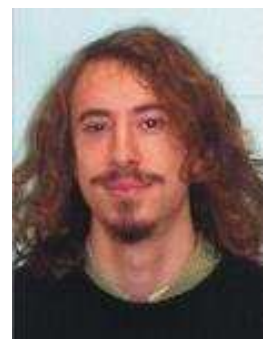

Vincent Crocher received a master's degree in electronics and computer science (2008) and is currently a $\mathrm{PhD}$ student at the University of Paris 6 since october 2008. He is working on orthosis control for upper limb rehabilitation, at the Institute of Intelligent Systems and Robotics.

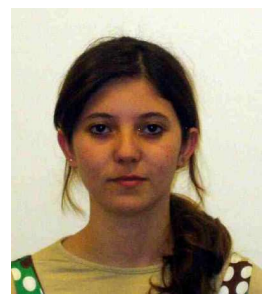

Amina Maiza is currently finishing her Master of Science degree in robotics at Polytech'Paris / Univ. P\&M Curie, France. She has been doing an internship at the Neurophysics and Physiology Laboratory, CNRS 8119, Paris, France and has been working on upper limb synergies and movement analysis.

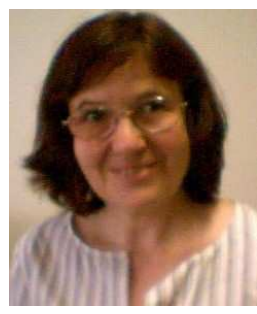

Dr. Agnès Roby-Brami is physician who performed her residency in Paris Hospitals (1977-1982). She received a MD degree from University Paris Descartes (1982) and MSc and $\mathrm{PhD}$ degrees and University Habilitation in Neurosciences from Pierre et Marie Curie University $(1982,1991,2001)$. She was appointed as a Research Associate at INSERM in 1983 and promoted to Research Director in 2005. A. Roby-Bramis first research interest was in the field of clinical neurophysiology after which she focused on upper limb motor control, disability and recovery in cerebral and spinal injured patients. A. Roby-Brami is the author or coauthor of 71 peer-reviewed academic publications, and 44 contributions to edited books. She is also actively involved in several French Academic Networks concerning research on disability.

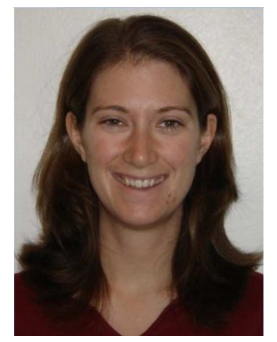

Dr Johanna VG Robertson is physiotherapist (Robert Gordons University, Aberdeen, UK, 1999) and has worked in several hospitals in the UK and France. She received MS and $\mathrm{PhD}$ degrees in Neurosciences from Pierre et Marie Curie University (2010). Her present position is Research Therapist, Raymond Poincar Hospital, Garches and research associate in the Neurophysics and Physiology Laboratory, CNRS 8119, Paris, France. Her scientific interest is devoted to increasing the understanding of upper limb motor synergies post-stroke to improve and adapt appropriate rehabilitation techniques. J. Robertson is the author or coauthor of 4 peer-reviewed academic publications, and 3 contributions to edited books.

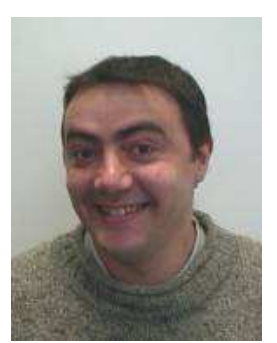

Guillaume Morel received a M.S. in electrical engineering (1990) and a $\mathrm{PhD}$ in mechanical engineering (1994), in Univ. P\&M Curie, France. After a postdoc at M.I.T. and a first assistant professorship in Strabsourg, he came back to Paris in 2001. Over these years, his reserach interests have been force feedback control and visual servoing of robots, with, for the last decade, a particular focus on medical applications. He now heads a multlidisciplinary group developing devices aimed at assisting gesture through the concept of comanipulation. 